\title{
On the theory of row-column designs
}

\section{Kenneth Graham Russell}

If an experiment is to be conducted in a heterogeneous medium, the experimenter may endeavour to reduce the experiment's inherent variability by dividing the medium into segments which are relatively homogeneous. If this requires it to be divided in two different directions, the medium may be considered to be partitioned into a number of rows and columns. Treatment is then applied to the experimental units in each homogeneous segment, and the resulting layout is known as a row-coluran design, or a design for the two-way elimination of heterogeneity. The theory of such designs is applicable to any design where the systematic variability is due to three distinct factors.

This thesis examines the theory of row-column designs. In two preliminary chapters, known results on estimability and orthogonality, and on the testing of hypotheses, are presented. All work is done under the assumption of the linear additive fixed-effects model.

In Chapter 4 the orthogonality of two factors after each has been adjusted for a third factor is defined, and a necessary and sufficient condition for its existence is obtained. The presence of adjusted orthogonality is shown to simplify the requirements for a factor to be connected.

Chapter 5 treats the estimability and connectedness of a factor of a row-column design. A method of determining the estimable contrasts of a factor's levels, and estimators of them, is presented. It is shown that, in certain cases, these estimators are the least squares estimators. The method of estimation is similar to that of Birnes, Dodge, and Seely [1].

Chapter 6 considers the introduction of a second blocking system into

Received 16 June 1977. Thesis submitted to the University of New South Wales, October 1976. Degree approved, June 1977. Supervisor: Dr J. A. Eccleston. 
balanced incomplete block designs to convert them to row-column designs. As such, they are a sub-type, $0: X B$, of $0: X T$ designs (using Pearce's notation [2], where $X$ represents orthogonality or the degree of balance). $0: X B$ designs are shown to be pairwise connected, and a necessary and sufficient condition is obtained for the overall connectedness of $0: X T$ designs. The $(M, S)$-optimality criterion is used to optimise $0: X B$ designs. M-optimal designs, which are obtained by use of systems of distinct representatives, are shown to be connected, and are categorised into three classes. Procedures are given for obtaining the $(M, S)$-optimal design in two of these classes. The third class requires trial-and-error methods, and the most efficient designs obtained are displayed.

The final chapter extends the results of Chapter 4 to the multi-factor case, and the concept of adjusted orthogonality is considered in complete generality. Classical orthogonality between pairs of factors is shown to be a particular case of adjusted orthogonality.

The appendix contains several APL computer programs for use with rowcolumn designs. Additionally, a proof is given that, for many parameter sets, all $0: X B$ designs are connected.

\section{References}

[1] David Birkes, Yadolah Dodge and Justus Seely, "Spanning sets for estimable contrasts in classification models", Ann. Statist. 4 (1976), 86-107.

[2] S.C. Pearce, "The use and classification of non-orthogonal designs", J. Roy. Statist. Soc. Ser. A 126 (1963), 353-369. 